Gynecologic and

Obstetric Investigation
Gynecol Obstet Invest 2012;74:151-156

DOI: $\underline{10.1159 / 000337713}$
Received: December 7, 2011

Accepted after revision: February 8, 2012

Published online: June 21, 2012

\title{
Postoperative Depot Medroxyprogesterone Acetate versus Continuous Oral Contraceptive Pills in the Treatment of Endometriosis-Associated Pain: A Randomized Comparative Trial
}

\author{
Sopon Cheewadhanaraks ${ }^{\mathrm{a}}$ Chainarong Choksuchat ${ }^{\mathrm{a}}$ Kriengsak Dhanaworavibul $^{\mathrm{a}}$ \\ Tippawan Liabsuetrakul ${ }^{b}$ \\ ${ }^{a}$ Department of Obstetrics and Gynecology, and ${ }^{\mathrm{b}}$ Epidemiology Unit, Faculty of Medicine, Prince of Songkla \\ University, Hat Yai, Thailand
}

\author{
Key Words \\ Depot medroxyprogesterone acetate - Endometriosis • \\ Oral contraceptives · Pain
}

\begin{abstract}
Background/Aim: To evaluate the efficacy and tolerability of postoperative depot medroxyprogesterone acetate (DMPA) versus postoperative continuous oral contraceptive (OC) pills in the treatment of endometriosis-associated pain. Methods: After a conservative surgery, 84 patients with symptomatic endometriosis were randomized to receive either intramuscular DMPA (150 mg) every 12 weeks for 24 weeks or continuous OC pills (ethinyl estradiol $0.03 \mathrm{mg}$ and gestodene $0.075 \mathrm{mg}$ ) daily for 24 weeks. At weeks 12 and 24 of the treatment phase, patients rated their satisfaction with treatment and reported pain improvement and adverse effects. Results: There was no significant difference in the percentages of patients who reported satisfaction between the DMPA group and the OC group at weeks 12 and 24 (92.9 vs. $90.5 \%$, and 92.9 vs. $88.1 \%$, respectively). The rates of withdrawal because of persistent pain or side effects in the two groups were similar. Pain scores improved significantly in both groups, but dysmenorrhea scores on a visual analog scale at week 24 were significantly higher in the OC group
\end{abstract}

than in the DMPA group ( $p=0.039)$. Conclusion: Both postoperative DMPA and postoperative OC pills for 24 weeks were found to be effective and acceptable options for treating endometriosis-associated pain.

Copyright $\odot 2012$ S. Karger AG, Basel

\section{Introduction}

Endometriosis is a chronic and recurrent disease which impairs quality of life and imposes significant costs in many countries [1]. Medical and conservative surgical treatments are the current options for symptomatic patients and for those who wish to preserve their fertility. A randomized, double-blind, placebo-controlled trial demonstrated that cyclic oral contraceptive (OC) pills are a useful option in the hormonal treatment of endometriosis [2]. In women with endometriosis-associated recurrent dysmenorrhea that does not respond to cyclic OC use, long-term reduction of pain and an appreciable degree of overall satisfaction with therapy can be obtained by continuous administration of a monophasic OC [3]. Postoperative continuous OC pills were demonstrated to be more effective than surgery plus placebo or surgery alone and were equally effective compared with

\section{KARGER}

Fax +4161306 1234

E-Mail karger@karger.ch

www.karger.com (c) 2012 S. Karger AG, Basel

$0378-7346 / 12 / 0742-0151 \$ 38.00 / 0$

Accessible online at:

www.karger.com/goi
Sopon Cheewadhanaraks, MD

Department of Obstetrics and Gynecology, Faculty of Medicine

Prince of Songkla University

Hat Yai, Songkla 90110 (Thailand)

Tel. +66 74451 201, E-Mail csophon@medicine.psu.ac.th 
surgery plus a gonadotropin-releasing hormone agonist in reducing endometriosis-associated pain $[4,5]$.

Among the various medical therapies, based on a more favorable profile in terms of safety, tolerability and cost, combined OCs and progestins should be considered as the first-line option, both as an alternative to surgery and as a postoperative adjuvant measure [6]. The efficacy and safety of intramuscular depot medroxyprogesterone acetate (DMPA) [7-11] and subcutaneous DMPA [12, 13] have been studied, and both regimens appear to be effective and safe for the treatment of endometriosis-associated pain. However, to the best of our knowledge, there is no study in English evaluating the efficacy and adverse effects of postoperative DMPA treatment. Subjects in previous studies [7-13] were women with recurrence of endometriosis-associated pain after previous conservative surgery or patients who had undergone a diagnostic laparoscopy. In the present study, we compared the benefits and side effects of two first-line drugs administered after conservative surgery in patients with symptomatic endometriosis. Our purpose was to evaluate the efficacy, safety and tolerability of postoperative intramuscular DMPA versus postoperative continuous OC pills in the treatment of pelvic pain in women with endometriosis.

\section{Materials and Methods}

This randomized, open-label, comparative study was approved by the institutional review board and conducted in our university hospital between September 2007 and October 2011. Eligible patients were premenopausal women from 18 to 40 years of age who had had endometriosis-associated pain for at least 6 months and did not wish to conceive in the next 18 months or more. Severity of dysmenorrhea, nonmenstrual pain and deep dyspareunia were evaluated using a $10-\mathrm{cm}$ visual analog scale (VAS) on which zero indicated absence of pain and 10 indicated unbearable pain, as well as a verbal rating scale (VRS). The VRS was modified from the pain measurement tool of Biberoglu and Behrman [14], which consists of scores $0,1,2$ and 3, as follows: dysmenorrhea score, $0=$ no pain, $1=$ no absence from work but decreased efficiency, $2=a b$ sence from work on less than 1 day per cycle, $3=$ absence from work on 1 day or more per cycle; nonmenstrual pain score, $0=$ no pain, 1 = occasional pain but no analgesic needed, $2=$ analgesic needed in some cycles, 3 = analgesic needed in every cycle; deep dyspareunia score, $0=$ no pain, $1=$ pain tolerated, $2=$ painful intercourse to point of interruption, 3 = avoidance of intercourse because of pain. All the women recruited for the study presented with a preoperative VAS score of 5 or more for at least one type of pain. Exclusion criteria were medical treatments for endometriosis other than non-steroid anti-inflammatory drugs within the previous 6 months, other pelvic pathology (adenomyosis, chronic pelvic inflammatory disease, submucous myoma uteri), known gastrointestinal, urologic and orthopedic diseases and contraindications to DMPA and OC pills.
A first surgical diagnosis of endometriosis was made and staged using the revised American Society for Reproductive Medicine classification [15]. Patients with minimal, mild and moderate endometriosis underwent conservative surgery via laparoscopy, and patients with severe disease via laparotomy. In patients with severe endometriosis, we could remove endometriotic lesions more thoroughly via laparotomy than via laparoscopy. Complete excision or coagulation of visible and palpable endometriotic lesions was performed unless a large bowel perforation was anticipated. Peritoneal endometriotic lesions were treated by bipolar electrocoagulation, adhesions by lysis, ovarian endometriomas by cystectomy and rectovaginal endometriosis by excision. No conversions to laparotomy were needed in patients undergoing laparoscopic surgery. Histopathologic examination reports were used to confirm the endometriotic nature of the lesions. After the subjects gave written informed consent to the study, treatment allocation was performed in accordance with a computergenerated randomization sequence with the use of numbered, opaque, sealed envelopes. Postoperative medical treatments were initiated within the first 5 days of a menstrual cycle. The DMPA group received intramuscular DMPA injections $(150 \mathrm{mg})$ at our hospital every 12 weeks for 24 weeks, and the OC group took continuous monophasic combined OC pills (ethinyl estradiol $0.03 \mathrm{mg}$ and gestodene $0.075 \mathrm{mg}$ ) daily for 24 weeks. If there was prolonged breakthrough bleeding for 7 days during the continuous OC administration, the women were advised to suspend treatment for 1 week [3]. The subjects were permitted to take acetaminophen when needed. The patients were asked to come for follow-up visits at weeks 12 and 24 of treatment. The OC pills were counted to assess compliance with the treatment, and their diaries were reviewed to evaluate bleeding patterns. Bleeding patterns were categorized into three groups: amenorrhea (no bleeding for at least 3 months), spotting (slight bleeding) and breakthrough bleeding (any other bleeding). Side effects were recorded, and pain improvement was measured by VRS and VAS. The patients rated their overall degree of satisfaction with treatment (very satisfied, satisfied, uncertain, dissatisfied, very dissatisfied). Pain during erratic bleeding was considered dysmenorrhea and pain without bleeding as nonmenstrual pain. Dysmenorrhea scores in women who were amenorrheic were counted as 0 on both scales. Patients who were very satisfied or satisfied with the treatment were classified as patients with satisfaction. Patients without satisfaction were those who were uncertain, dissatisfied or very dissatisfied, who withdrew due to persistent pain or adverse side effects, were lost to follow-up and who violated the protocol. The primary outcome measure was the percentage of patients with satisfaction with the treatment at weeks 12 and 24, with other outcome measures being the rate of withdrawal due to persistent pain or adverse side effects and reduction in pain scores. At the end of the medical treatment phase, a transvaginal ultrasonography was performed to assess ovarian endometrioma(s).

According to the results of an intention-to-treat analysis in our pilot study, the percentage of patients who were satisfied or very satisfied with the postoperative continuous OC therapy was $57 \%$. Of the 14 patients, 8 were satisfied or very satisfied, 1 had persistent pain, 1 withdrew due to adverse effects, 2 violated the protocol and 2 were lost to follow-up. In our previous report [10], we noted that $73.2 \%$ of patients were satisfied with intramuscular DMPA injections for 24 weeks after a diagnostic laparoscopy. In this trial, the cytoreductive effect of the surgery before DMPA 
treatment might have increased satisfaction with treatment, so we expected $85 \%$ of patients to be satisfied or very satisfied with the postoperative DMPA treatment. A sample size of 40 patients in each group would give the study an $80 \%$ chance to detect a difference of $28 \%$ between the two groups at an overall significance level of 5\%. The statistical significance of the difference in the proportion of patients with satisfaction, pain scores by VRS and stages was compared with $\chi^{2}$ test or Fisher's exact test. The pain scores by VAS, age, body mass index and revised American Society for Reproductive Medicine scores were evaluated with the Student $t$ test or rank sum test, as appropriate. All statistical tests were two-sided, and probability values less than 0.05 were considered significant.

\section{Results}

There were 84 patients randomized, and all of them received the study medication (fig. 1). The demographics and pretreatment characteristics of the two groups were similar (table 1). The percentages of patients with residual disease at cul-de-sac after the surgery in the two groups were not significantly different 3 of 42 patients (7.1\%) in the DMPA group and 2 of 42 (4.8\%) in the OC group]. At weeks 12 and 24 of the treatment phase, there was no statistically significant difference in the percentages of patients with satisfaction between the DMPA group and the OC group [39 of 42 subjects (92.9\%) vs. 38 of 42 (90.5\%), and 39 of $42(92.9 \%)$ vs. 37 of 42 (88.1\%), respectively]. The rates of withdrawal because of persistent pain in the two groups were not different [ 1 of 42 participants (2.4\%) in the DMPA group and 3 of 42 (7.1\%) in the OC group]. The pretreatment scores of the three types of pain assessed by VRS and VAS in the two groups (table 2) were all similar. At weeks 12 and 24, dysmenorrhea scores, dyspareunia scores and nonmenstrual pain scores measured by VRS and VAS (table 2) improved significantly in both groups. However, dysmenorrhea scores by VAS at week 24 in the OC group were significantly higher than those in the DMPA group ( $p=0.039$; table 2 ). There was no statistically significant difference between the proportions of patients who needed acetaminophen [8 of 42 participants (19.0\%) in the DMPA group and 12 of 42 (28.6\%) in the OC group].

The percentages of patients who left the study because of side effects were similar [ 2 of 42 subjects $(4.8 \%)$ in the DMPA group and 1 of $42(2.4 \%)$ in the OC group]. The main side effects causing withdrawal from the study were weight gain in the DMPA group and breakthrough bleeding in the OC group. Frequent adverse effects experienced were oily skin (15 of 39 patients; $38.5 \%$ ) and irritability $(12$ of $39 ; 30.8 \%)$ in the DMPA group and mastalgia

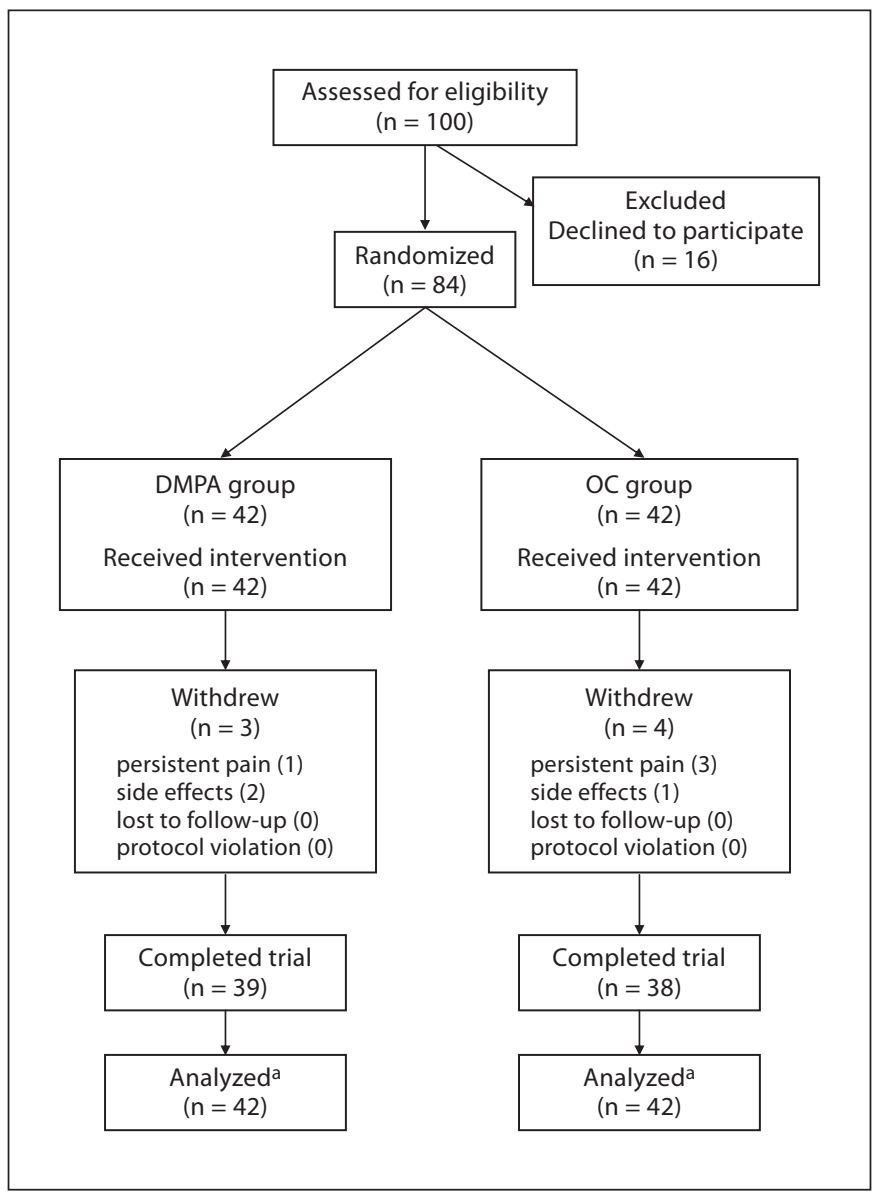

Fig. 1. The flow of patients through the study. ${ }^{\text {a }}$ Intention-to-treat analysis.

Table 1. Patient demographics and pretreatment characteristics

\begin{tabular}{lcc}
\hline Characteristic & $\begin{array}{l}\text { DMPA group } \\
(\mathrm{n}=42)\end{array}$ & $\begin{array}{l}\text { OC group } \\
(\mathrm{n}=42)\end{array}$ \\
\hline Age, years & $31.9 \pm 5.5$ & $30.5 \pm 5.4$ \\
BMI & $21.8 \pm 4.3$ & $21.0 \pm 3.7$ \\
rASRM score & $19.5(4-43.5)$ & $28(5.2-51)$ \\
Stage & & \\
$\quad$ Minimal & $12(28.6)$ & $11(26.2)$ \\
$\quad$ Mild & $8(19.0)$ & $7(16.7)$ \\
$\quad$ Moderate & $7(16.7)$ & $6(14.3)$ \\
$\quad$ Severe & $15(35.7)$ & $18(42.9)$ \\
Unilateral endometrioma & $12(28.6)$ & $12(28.6)$ \\
Bilateral endometrioma & $6(14.3)$ & $6(14.3)$ \\
Complete cul-de-sac obliteration & $11(26.2)$ & $14(33.3)$ \\
\hline
\end{tabular}

Values are shown as means $\pm \mathrm{SD}$, medians (interquartile range) or numbers of patients (percentage), as appropriate. $p$ values: not significant. BMI = Body mass index; rASRM = revised American Society for Reproductive Medicine classification. 
Table 2. Pain scores before, during and at the end of treatment

\begin{tabular}{|c|c|c|c|c|c|c|}
\hline \multirow[t]{2}{*}{ Scale } & \multicolumn{2}{|l|}{ Dysmenorrhea } & \multicolumn{2}{|c|}{ Deep dyspareunia } & \multicolumn{2}{|c|}{ Nonmenstrual pain } \\
\hline & DMPA group & OC group & DMPA group & OC group & DMPA group & OC group \\
\hline \multicolumn{7}{|c|}{ Verbal rating ${ }^{\mathrm{a}}$} \\
\hline \multicolumn{7}{|c|}{ Baseline } \\
\hline 0 & 2 & 3 & 7 & 6 & 19 & 18 \\
\hline 1 & 8 & 4 & 7 & 3 & 6 & 8 \\
\hline 2 & 10 & 11 & 2 & 5 & 7 & 8 \\
\hline 3 & 22 & 24 & 4 & 4 & 10 & 8 \\
\hline \multicolumn{7}{|l|}{ Week 12} \\
\hline 0 & 32 & 26 & 13 & 14 & 35 & 30 \\
\hline 1 & 6 & 11 & 3 & 4 & 5 & 11 \\
\hline 2 & 0 & 2 & 1 & 0 & 0 & 1 \\
\hline 3 & 2 & 3 & 1 & 0 & 0 & 0 \\
\hline \multicolumn{7}{|l|}{ Week 24} \\
\hline 0 & 32 & 24 & 12 & 13 & 30 & 28 \\
\hline 1 & 7 & 14 & 4 & 3 & 7 & 10 \\
\hline 2 & 0 & 0 & 1 & 0 & 2 & 0 \\
\hline 3 & 0 & 0 & 0 & 0 & 0 & 0 \\
\hline \multicolumn{7}{|c|}{ Visual analog } \\
\hline Baseline & $9(7-10)$ & $8.2(7-10)$ & $3(0-5)$ & $4.5(0-7)$ & $2.5(0-6.8)$ & $2(0-6.4)$ \\
\hline Week 12 & $0(0-0)$ & $0(0-2.8)$ & $0(0-2.2)$ & $0(0-0)$ & $0(0-0)$ & $0(0-0.6)$ \\
\hline Week 24 & $0(0-0)^{*}$ & $0(0-3)^{*}$ & $0(0-2)$ & $0(0-0)$ & $0(0-0)$ & $0(0-0.4)$ \\
\hline
\end{tabular}

(21 of $42 ; 50.0 \%)$ and nausea (15 of $42 ; 35.7 \%)$ in the OC group. Adverse side effects were generally well tolerated by the majority of patients in both groups. At the end of the study intervention, the rates of amenorrhea, spotting and breakthrough bleeding were $17.9 \%$ (7 of 39 patients), $71.8 \%$ (28 of 39) and $10.3 \%$ (4 of 39), respectively, in the DMPA group, and $7.9 \%$ (3 of 38), 63.2\% (24 of 38) and $28.9 \%$ (11 of 38), respectively, in the OC group. Thirtyseven patients $(88.1 \%)$ took at least $90 \%$ of the OC pills according to the regimen. Five patients (11.9\%) in the OC group had to have 1-week therapy suspensions for prolonged breakthrough bleeding. At week 24, no subjects showed signs of ovarian endometriomas.

\section{Discussion}

Although the patients in the OC group reported higher dysmenorrhea scores overall than those in the DMPA group, there was no statistically significant difference in the percentages of patients with satisfaction between the two groups at weeks 12 and 24 . The rates of withdrawal because of persistent pain or adverse side effects were not different between the two groups. Patient satisfaction was selected to be the main outcome measure of the present study, as an indicator for both pain relief and tolerance to drug side effects simultaneously. Dysmenorrhea experienced by the subjects in the OC group might not have been severe enough to influence their satisfaction ratings. We did not incorporate a follow-up phase after the end of the hormonal treatment in our study since medical therapy is symptomatic and pain relapse at treatment suspension is very likely to occur sooner or later [16]. Vercellini et al. [17] recommended that after first-line surgery, women should be advised to use an OC until pregnancy is desired. Our findings indicate that DMPA following conservative surgery is another option for patients with endometriosis-associated pain who do not desire pregnancy in the near future.

A methodological drawback of this study was the open-label design. A double-blind design would have been more reliable but was not feasible due to a prohibition against placebo injections in our hospital. However, the strengths of our clinical trial include the zero 
rates of protocol violation and loss to follow-up, the use of two pain assessment tools (VRS and VAS) and the choice of two first-line drugs for endometriosis-associated pain. DMPA as well as continuous OC pills are both inexpensive, have a more limited metabolic impact than other hormonal drugs and may be used on a longterm basis.

DMPA inhibits a luteinizing hormone surge and prevents ovulation. Endogenous estradiol levels vary; however, mean levels are lower than those of normally cycling women [18]. Inhibition of ovulation and low levels of estradiol result in a suboptimal environment for ectopic implants to grow and reduce the associated pain symptoms. Nevertheless, there are many problems associated with the use of DMPA. Firstly, in comparison with OC, erratic bleeding is much more difficult to manage and may persist for weeks. This may be upsetting and influence satisfaction with treatment. Secondly, if a side effect or an adverse event ensues, treatment with DMPA cannot be discontinued as the effect may last for 6-8 months after an intramuscular injection. Thirdly, for the same reason, DMPA may not be a reasonable choice for women wanting a pregnancy in the immediate future, as ovulation may be inhibited for up to 12 months. Fourthly, postoperative medical treatment is intended for prolonged periods of time, not only a few months. From this perspective, the effect of DMPA on bone density is of major concern and must not be dismissed. A large population-based case-control study [19] suggests that the use of DMPA is associated with a slightly increased risk of fractures, especially current use of 10 or more DMPA prescriptions compared with nonuse (adjusted odds ratio 1.54, 95\% confidence interval 1.33-1.78). OCs used continuously do not share the above disadvantages.

Combined OC pills act via many mechanisms in the treatment of endometriosis. Continuous OC pills prevent ovulation and suppress ovarian estradiol production. Also, OC pills induce decidualization of endometriotic lesions, followed by atrophy, suppress cell proliferation and enhance apoptosis of eutopic endometrium [20] and decrease aromatase expression in eutopic endometrial tissue [21]. Our OC pill regimen was demonstrated to be safe by Machado et al. [22]. In their study, the continuous use of the combination of ethinyl estradiol $0.03 \mathrm{mg}$ and gestodene $0.075 \mathrm{mg}$ for 24 weeks was associated with metabolic changes similar to those observed in patients using the pill with a monthly pill-free interval, with no changes in blood pressure or weight.

Treatment of Endometriosis-Associated Pain
There are 3 randomized trials in PubMed comparing postsurgical continuous combined OC pills with surgery plus placebo or surgery plus other hormonal drugs. Sesti et al. [4] reported that postoperative continuous OC pills (ethinyl estradiol $0.03 \mathrm{mg}$ and gestodene $0.075 \mathrm{mg}$ daily) for 6 months were more effective than surgery plus placebo and were equally effective compared with surgery plus 6 months of a gonadotropin-releasing hormone agonist in reducing dysmenorrhea and nonmenstrual pain. In the study of Seracchioli et al. [5], a significant reduction in dysmenorrhea was found in patients who underwent laparoscopic excision for symptomatic ovarian endometrioma and received postoperative continuous OC pills (ethinyl estradiol $0.02 \mathrm{mg}$ and gestodene $0.075 \mathrm{mg}$ daily) for 24 months versus those who received postsurgical cyclic OC pills and those who received no postoperative medical treatment. Our findings that $88.1 \%$ of our subjects were satisfied or very satisfied with their treatment confirm the efficacy and tolerability of 24 weeks of postoperative continuous OC pills. In a recent small study by Muzii et al. [23], continuous and cyclic administration of OC pills (ethinyl estradiol $0.02 \mathrm{mg}$ and desogestrel $0.15 \mathrm{mg}$ daily) for 6 months after operative laparoscopy to treat ovarian endometrioma(s) associated with pain were equally effective in prevention of recurrence of pain. However, the continuous regimen was associated with a significantly higher rate of adverse effects (breakthrough bleeding in particular), leading to discontinuation of treatment in $41 \%$ of patients. The rate of withdrawal due to adverse effects in our study was only $2.4 \%$. The active ingredients of our OC pills were different from those in their study, which might account for the lower rate of withdrawal in our study.

In conclusion, both postoperative DMPA and postoperative continuous OC pills for 24 weeks were demonstrated to be effective and acceptable options for treating endometriosis-associated pain in women who did not wish to get pregnant in the near future; however, dysmenorrhea scores were higher in the OC group than in the DMPA group.

\section{Acknowledgment}

The authors thank the Prince of Songkla University, Thailand, for full financial support of this study. 


\section{References}

-1 Simoens S, Hummelshoj L, Dunselman G, Brandes I, Dirksen C, D'Hooghe T; EndoCost Consortium: Endometriosis cost assessment (the EndoCost study): a cost-of-illness study protocol. Gynecol Obstet Invest 2011;71:170-176.

-2 Harada T, Momoeda M, Taketani Y, Hoshiai $\mathrm{H}$, Terakawa N: Low-dose oral contraceptive pill for dysmenorrhea associated with endometriosis: a placebo-controlled, doubleblind, randomized trial. Fertil Steril 2008; 90:1583-1588.

- 3 Vercellini P, Frontino G, De Giorgi O, Pietropaolo G, Pasin R, Crosignani PG: Continuous use of an oral contraceptive for endometriosis-associated recurrent dysmenorrhea that does not respond to a cyclic pill regimen. Fertil Steril 2003;80:560-563.

-4 Sesti F, Pietropolli A, Capozzolo T, Broccoli P, Pierangeli S, Bollea MR, et al: Hormonal suppression treatment or dietary therapy versus placebo in the control of painful symptoms after conservative surgery for endometriosis stage III-IV. A randomized comparative trial. Fertil Steril 2007;88:15411547.

5 Seracchioli R, Mabrouk M, Frascà C, Manuzzi L, Savelli L, Venturoli S: Long-term oral contraceptive pills and postoperative pain management after laparoscopic excision of ovarian endometrioma: a randomized controlled trial. Fertil Steril 2010;94: 464-471.

6 Vercellini P, Somigliana E, Viganò P, Abbiati A, Daguati R, Crosignani PG: Endometriosis: current and future medical therapies. Best Pract Res Clin Obstet Gynaecol 2008; 22:275-306.

7 Vercellini P, De Giorgi O, Oldani S, Cortesi I, Panazza S, Crosignani PG: Depot medroxyprogesterone acetate versus an oral contraceptive combined with very-low-dose danazol for long-term treatment of pelvic pain associated with endometriosis. Am J Obstet Gynecol 1996;175:396-401.
8 Arowojolu AO: Treatment of endometriosis with depot medroxyprogesterone acetate: a preliminary experience. Afr J Med Med Sci 2000;29:55-58.

-9 Walch K, Unfried G, Huber J, Kurz C, van Trotsenburg M, Pernicka E, et al: Implanon versus medroxyprogesterone acetate: effects on pain scores in patients with symptomatic endometriosis - a pilot study. Contraception 2009;79:29-34.

10 Cheewadhanaraks S, Peeyananjarassri K, Choksuchat C, Dhanaworavibul K, Choobun $\mathrm{T}$, Bunyapipat S: Interval of injections of intramuscular depot medroxyprogesterone acetate in the long-term treatment of endometriosis-associated pain: a randomized comparative trial. Gynecol Obstet Invest 2009;68:116-121.

11 Wong AY, Tang LC, Chin RK: Levonorgestrel-releasing intrauterine system (Mirena) and depot medroxyprogesterone acetate (Depoprovera) as long-term maintenance therapy for patients with moderate and severe endometriosis: a randomized controlled trial. Aust NZ J Obstet Gynaecol 2010;50: 273-279.

12 Crosignani PG, Luciano A, Ray A, Bergqvist A: Subcutaneous depot medroxyprogesterone acetate versus leuprolide acetate in the treatment of endometriosis-associated pain. Hum Reprod 2006;21:248-256.

13 Schlaff WD, Carson SA, Luciano A, Ross D, Bergqvist A: Subcutaneous injection of depot medroxyprogesterone acetate compared with leuprolide acetate in the treatment of endometriosis-associated pain. Fertil Steril 2006;85:314-325.

14 Biberoglu KO, Behrman SJ: Dosage aspects of danazol therapy in endometriosis: shortterm and long-term effectiveness. Am J Obstet Gynecol 1981;139:645-654.
15 Revised American Society for Reproductive Medicine classification of endometriosis: 1996. Fertil Steril 1997;67:817-821.

16 Vercellini P, Cortesi I, Crosignani PG: Progestins for symptomatic endometriosis: a critical analysis of the evidence. Fertil Steril 1997;68:393-401.

17 Vercellini P, Somigliana E, Viganò P, De Matteis S, Barbara G, Fedele L: Post-operative endometriosis recurrence: a plea for prevention based on pathogenetic, epidemiological and clinical evidence. Reprod Biomed Online 2010;21:259-265.

18 Mishell DR Jr: Pharmacokinetics of depot medroxyprogesterone acetate contraception. J Reprod Med 1996;41(5 suppl):381-390.

-19 Meier C, Brauchli YB, Jick SS, Kraenzlin ME, Meier CR: Use of depot medroxyprogesterone acetate and fracture risk. J Clin Endocrinol Metab 2010;95:4909-4916.

20 Meresman GF, Augé L, Barañao RI, Lombardi E, Tesone M, Sueldo C: Oral contraceptives suppress cell proliferation and enhance apoptosis of eutopic endometrial tissue from patients with endometriosis. Fertil Steril 2002;77:1141-1147.

21 Maia H Jr, Casoy J, Correia T, Freitas LA, Pimentel K, Athayde C: The effect of oral contraceptives on aromatase expression in the eutopic endometrium of patients with endometriosis. Gynecol Endocrinol 2008;24:123128 .

22 Machado RB, Fabrini P, Cruz AM, Maia E, da Cunha Bastos A: Clinical and metabolic aspects of the continuous use of a contraceptive association of ethinyl estradiol $(30 \mathrm{mi}-$ crog) and gestodene (75 microg). Contraception 2004;70:365-370.

23 Muzii L, Maneschi F, Marana R, Porpora MG, Zupi E, Bellati F, et al: Oral estroprogestins after laparoscopic surgery to excise endometriomas: continuous or cyclic administration? Results of a multicenter randomized study. J Minim Invasive Gynecol 2011;18: 173-178. 